\title{
A review on roselle fiber and its composites
}

\begin{abstract}
Recently, due to increasing environmental concerns, scientists and researchers have been replacing synthetic fibers with natural fibers as the main component in composites. Roselle is one suitable natural fiber. The plantôs history, physical description, cultural methods, and usages were explained to further understand this potential plant. Based on a literature review, it is clear that extensive research has been performed on the fiber extraction methods, morphology, properties, and possible surface treatments of roselle to enhance its properties in the manufacturing of natural-fiber-reinforced polymer composites; however, no review has covered the properties and applications of roselle fibers in detail. Therefore, a review was performed on roselle to determine its potential to enable a better selection of materials to produce composites for potential applications in the construction and building, automotive, and aerospace industries.
\end{abstract}

Keyword: Applications; Mechanical properties; Morphology properties; Roselle fiber 